\title{
A radiographic assessment of the correlation between the calcification stages of the mandibular second molar and the middle phalanx of the third finger of 9-16 years old children
}

\author{
Mitul Joshi* (1D, Dinesh Rao, Shubha A.B., Sunil Panwar and Sachin Franklin
}

\begin{abstract}
Background: Hand-wrist radiographs and orthopantomographs are routinely used for assessing skeletal and dental maturation and analyzing skeletal morphology and growth patterns. Though minimal radiation is associated with a hand-wrist radiograph, it would be superlative to assess the growth stage without an additional radiograph.

Aim: To assess the correlation between the calcification stages of mandibular second molar and the modified middle phalanx of the third finger (modified MP3) of 9 to 16 year old children.

Design: In this cross-sectional observational study we assessed the dental calcification of the mandibular second molar on orthopantomograms using Demirjian's tooth developmental stages and skeletal maturity of the middle phalanx of the third finger on hand-wrist radiographs using modified MP3 method proposed by Rajagopal and Kansal (J Clin Orthod 36(7):398, 2002) of 100 children (48 boys and 52 girls), aged between 9 and 16 years.

Results: A strong association was found between calcification stages of mandibular right second molar and the developmental stages of the modified middle phalanx of the third finger of right hand in both the gender. Spearman rank order correlation coefficients between the developmental stages of the modified MP3 of hand and the developmental stages of the mandibular second molar and found to be statistically significant positive in males (0.62) and females (0.41) when Demirjian's Index (DI) and modified middle phalanx of the third finger stages were compared.

Conclusion: There was a good concordance between the stages of modified middle phalanx of the third finger and the Demirjian's tooth developmental stages. Demirjian's second molar calcification stages can be a better choice for predicting skeletal maturity because of its simplicity and reliability.
\end{abstract}

Keywords: Dental maturation, Growth prediction, Modified MP3, Mandibular second molar

\section{Background}

Children mature as they grow. Measures of growth are commonly interpreted in relation to levels of maturity in some parts of the skeleton, which will influence further clinical management. The changes associated with maturation are evident in many parts of the body including the craniofacial skeleton and the dentition, during the process of growth and development of any somatic

\footnotetext{
* Correspondence: mituljoshi87@gmail.com; drmitul91@gmail.com Department of Pediatric Dentistry, Pacific Dental College and Hospital, Udaipur, Rajasthan State 313024, India
}

structures, time plays a crucial role in determining the final morphological and dimensional results (Baccetti et al. 2005). In growing individuals, orthodontic treatment depends on skeletal growth. Orthodontic diagnosis and treatment planning for growing children must involve growth prediction, especially in the treatment of skeletal problems (Hegde et al. 2014). Hand-wrist radiographs are made routinely as a supplemental diagnostic aid to other essential diagnostic radiographs such as intra-oral periapical radiographs (IOPA), orthopantomogram (OPG), and lateral cephalograms in order to measure 
skeletal growth. The classical and most widely used method for skeletal age evaluation is the highly reliable analysis of the radiograph of the hand-wrist bone. The validity of the hand-wrist bone analysis has been confirmed by numerous studies (Reddy et al. 2014; Flores-Mir et al. 2006; Grave and Brown 1976; Houston et al. 1979).

Fishman (Fishman 1982) developed a system of hand-wrist skeletal maturation indicators (SMI), using four stages of bone maturation at six anatomic sites on the hand and the wrist. But the disadvantages of these routine methods of skeletal maturity assessments are that they required elaborate equipment, expensive and the higher radiation dose and exposure time.

Many studies (Hegde et al. 2014; Reddy et al. 2014; Vijayashree et al. 2014) have shown that a strong association exists between skeletal maturity and dental calcification stages. Generally, dental development can be assessed by either the phase of tooth eruption or the stage of tooth calcification, with the latter being more reliable. The ability to assess skeletal maturity by the stages of mandibular second molar calcification through the examination of a panoramic radiograph would offer an advantage over the conventional hand-wrist radiographic method. No additional exposure to radiation would be necessary if the assessment of skeletal maturity were performed through routine radiographs, keeping in mind the 'As Low as Reasonably Achievable' (ALARA) principle (Kumar et al. 2011).

Hence the present study was done to provide a simple and practical method for assessing skeletal maturity using orthopantomographs, and to compare the eight developmental stages of the right mandibular second molar with that of six developmental stages of modified MP3 of the right hand. Further, to find out if any correlation exists, and to determine, if the developmental stages of the mandibular second molar alone can be used as a reliable indicator for assessment of skeletal maturity.

\section{Methods}

In this retrospective, cross-sectional observational study with a study sample selected using a convenience sampling method, carried out at the Pacific Dental College and Hospital, Udaipur, Rajasthan, India. One hundred healthy children ( 48 boys, 52 girls) of Indian origin and nationality, aged 9-16 years, having a complete mandibular permanent dentition, erupted or otherwise, who visited the Pacific Dental College and Hospital, during the period between July 2014 and June 2016 who required orthopantomograms and hand-wrist radiographs prior to correction of malaligned teeth were selected for the study. The prior permission obtained from the Department of Radiology for collecting radiographs in the study. The patients and parental consent was signed by the concerned authorities of Department Oral and Maxillofacial Radiology before the radiographs were made. Patients/Parents/Guardians had signed an agreement with the dental institution that dental records and radiographs could be used for future research and educational purposes without the possibility of personal identification.

The chronological age of the children in the study was determined from their date of birth mentioned in the respective case records. Radiographs of subjects with muscular dystrophy, congenital abnormalities affecting growth and development, traumatic lesions of the jaw or the hand and wrist, and other systemic conditions were excluded from the study. The present study assessed the dental calcification of the right mandibular second molar on orthopantomographs and skeletal maturity of the middle phalanx of the third finger on hand-wrist radiographs of the right hand.

Selection of the subjects was based on the following criteria:

i. The subjects were Rajasthan origin, well-nourished and free of any known serious illness.

ii. The subjects had undergone neither previous orthodontic treatment nor extraction of any permanent teeth.

iii. The subjects had normal dental conditions, for example, no impaction or transposition of teeth.

iv. The subjects had no previous history of trauma or injury to the face and the hand and wrist regions.

\section{Evaluation of dental maturity on panoramic radiographs}

In this study, the mandibular second molar was used as a sample for estimation of dental maturity on orthopantomograms. Tooth calcification was rated according to the index described by Demirjian et al. (Demirjian et al. 1973), in which one of eight stages of calcification (A to H) was assigned to the tooth (Fig. 1).

\section{Evaluation of modified MP3 stages on hand wrist radiographs}

The middle finger radiographs were evaluated according to the five stages of ossification described by Hagg and Taranger (Hägg and Taranger 1982) and later modified by adding MP3-HI stage by Rajagopal and Kansal (Rajagopal and Kansal 2002). This system uses six stages of bone maturation (Fig. 2).

All statistical analyses and data management were performed using the Statistical Package for Social Sciences 19.0 (SPSS Inc., Chicago, IL, USA) for Windows and MS-Excel (Microsoft Office 2010). Analyses were made for each gender and age group, and for the total sample. 


\section{Results}

In the present study a statistically significant positive association $(P<0.001)$ was found between DI and modified MP3 for both gender (Table 1). Association between the calcification stages of the mandibular right second molar and the modified MP3 of the right hand for female and male subject was found to be statistically highly significant $(\mathrm{P}<0.001)$ for both the gender, (Tables 2 and 3). D stage of the mandibular second molar correlated with modified MP3 F stages indicating the starting of the pubertal growth, Subjects with E, F, and G stage of the mandibular second molar was found to be associated with modified MP3 FG, G, and HI stages, which indicates that the completion of quarter to half of the pubertal growth (Figs. 3 and 4). G stage of the mandibular second molar correlated with modified MP3 I stage, which indicates minimal or no pubertal growth. Spearman rank order correlation coefficients was found between developmental stages of the modified MP3 of hand and the developmental stages of the mandibular second molar, which was found to be statistically significant among males (0.62) and females (0.41), when DI and modified MP3 stages were compared (Table 4).

\section{Discussion}

Assessment of the patient's puberty and developmental events is one of the most basic and important elements in orthodontic treatment. Information regarding the pubertal growth spurt of the patients plays a significant role in the diagnosis, objectives, and selection of the treatment method (Krailassiri et al. 2002). The use of hand-wrist radiographs has been advocated in order to assess the skeletal age of individuals. Several human growth studies (Flores-Mir et al. 2006; Houston et al. 1979; García-Fernandez et al. 1998; Uysal et al. 2004) have shown that the timing of the pubertal growth of the craniofacial region is closely related to specific ossification events and stages observed in the hand-wrist area

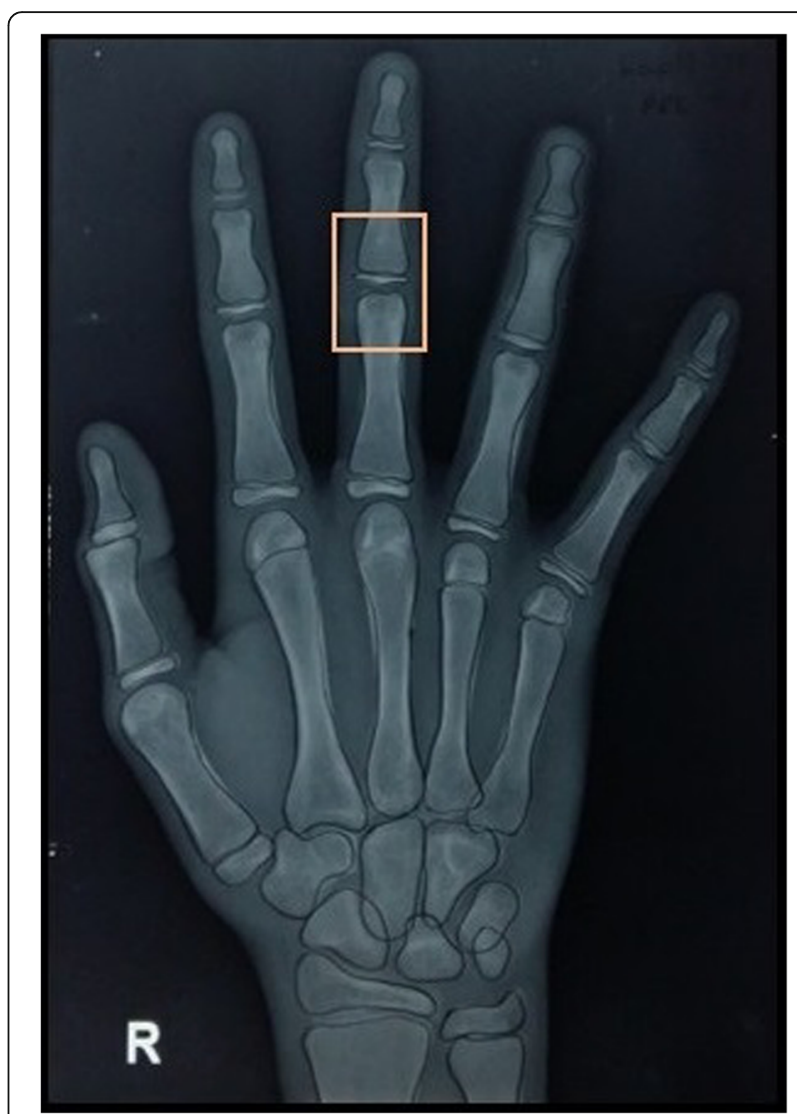

Fig. 2 Hand wrist radiograph of a subject showing modified MP3 stage of right hand

of the skeleton, therefore, hand-wrist radiographs have been proved to be a valuable diagnostic tool in orthodontics. However, these radiographs can cause unnecessary exposure to the patient. Thus the ALARA principle is important especially for children and young adults, as high radiation methods are not advised to be used frequently for the assessment of growth. The ease of distinguishing the stages of dental development and the

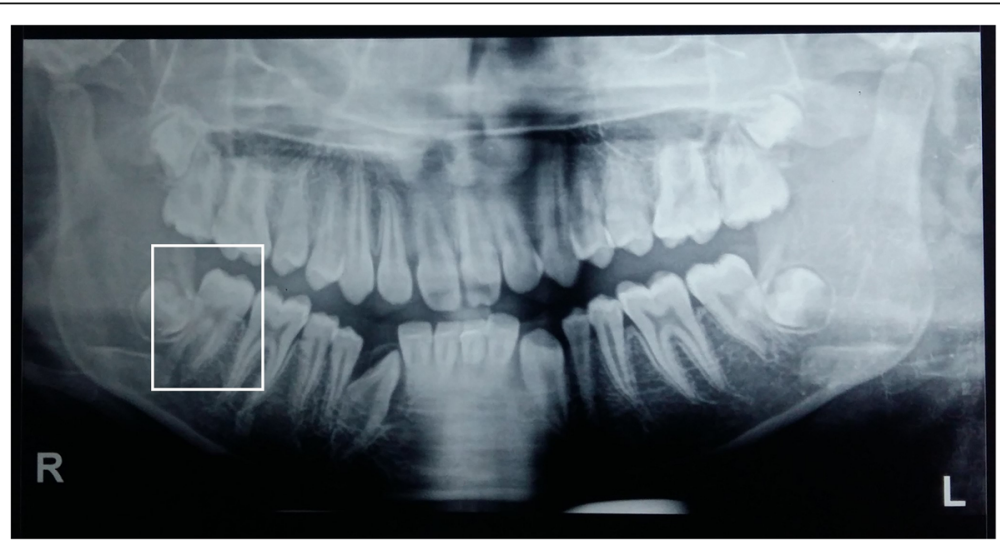

Fig. 1 Orthopentomograph of a subject showing calcification stage of right mandibular second molar 
Table 1 Comparison between DI and modified MP3 (both gender)

\begin{tabular}{llllllll}
\hline MP3 & DI & & & & & & $P$ value \\
\cline { 2 - 6 } & D & E & F & G & H & Total & \\
\hline F & 8 & 1 & 2 & 1 & 0 & 12 & $<0.0001(\mathrm{HS})$ \\
FG & 2 & 15 & 11 & 13 & 0 & 41 & \\
G & 1 & 0 & 2 & 7 & 0 & 10 & \\
H & 0 & 0 & 0 & 2 & 0 & 2 & \\
HI & 0 & 0 & 3 & 12 & 4 & 19 & \\
I & 0 & 3 & 4 & 4 & 5 & 16 & \\
Total & 11 & 19 & 22 & 39 & 9 & 100 &
\end{tabular}

Chi Square: 83.200. DF: 20. $P$ value is $<0.0001$

DI Demirjian's Index, MP3 middle phalanx of the third finger

availability of panoramic radiographs are practical reasons for attempting to assess the physiologic maturity without resorting to hand-wrist or lateral cephalometric radiographs (Krailassiri et al. 2002).

In previous radiographic studies, authors have used either mandibular third molars (Engström et al. 1983), premolars (Şahin Sağlam and Gazilerli 2002), canine (Yadav et al. 2017) or maxillary canine (Kumar et al. 2017) for the assessment of skeletal maturation, which exhibited certain drawbacks. Root formation and apex closure of canines and premolars usually completes by the age of 12 to 14 years, however most of the children exhibit active growth up to the age of 16 to 17 years. Third molars, on the other hand, are the most commonly missing teeth in the human dentition, making them unreliable for the age assessment. The current radiographic study, which has been conducted to evaluate the reliability of different developmental stages of mandibular second molars as an indicator of maturity. Mandibular second molar tooth offers an advantage over other teeth because of its developmental stages, which tends to continue over a longer period and later age. Apex closure of mandibular second molar generally extends up to the age of 16 years in normal children, which makes it more reliable in the assessment of growth. In the present study, mandibular second molar has been taken as a sample instead of maxillary molar and canine in order to eliminate the errors of estimation caused by roots of these teeth, which can overlap with the anatomic structures such as palate, inferior border of zygomatic arch, maxillary sinus septum etc. (Vijayashree et al. 2014).

It has long been opposed that dental eruption, which is the most conspicuous and easily determined indicator of dental maturation, is much more variable in its timing than skeletal maturation (Nolla 1960; Van der Linden et al. 1979). According to Nolla (Nolla 1960), dental eruption has also been reported to be more variable than the calcification sequence in the dentition. Dental eruption is a fleeting event that is under greater environmental influence (Demirjian et al. 1973). In the present study, calcification stages of teeth, rather than eruption, were preferred because tooth formation is proposed as a more reliable criterion for determining dental maturation (Nolla 1960). Therefore, the dental maturity assessment stages of Demirjian et al. (Demirjian et al. 1973), were used. This method also shows high accuracy when applied to Indian populations (Rai et al. 2008).

Since the system of evaluating hand wrist radiograph has been found to be valid in both clinical and research situations, include eleven discrete adolescent skeletal maturational indicators, covering the entire period of adolescent development, are found in hand wrist, and seven, out of these eleven discrete, occur in the third finger. Therefore the current study utilized the use of the middle phalanx of third finger of the right hand. Developmental changes in the middle phalanx of the third finger has been used to study the skeletal maturity development, as they follow the pubertal growth spurt from the onset to the end (García-Fernandez et al. 1998). Keeping in mind the ALARA principle, no additional exposure to radiation would be necessary if skeletal maturity can be assessed through routinely taken radiographs. The high degree of clarity and availability of the intra-oral periapical radiographs, the ease with which one can interpret the modified MP3 skeletal maturity stages, the low cost of IOPA films and the minimal

Table 2 Stages of modified MP3 compared with the stages of DI (female)

\begin{tabular}{|c|c|c|c|c|c|c|c|c|c|c|c|c|c|}
\hline \multirow{2}{*}{$\begin{array}{l}\text { DI } \\
\text { MP3 }\end{array}$} & \multicolumn{2}{|l|}{$\mathrm{D}$} & \multicolumn{2}{|l|}{ E } & \multicolumn{2}{|l|}{ F } & \multicolumn{2}{|l|}{ G } & \multicolumn{2}{|l|}{$\mathrm{H}$} & \multicolumn{2}{|c|}{ Total } & \multirow[t]{2}{*}{$P$ value } \\
\hline & No. & $\%$ & No. & $\%$ & No. & $\%$ & No. & $\%$ & No. & $\%$ & No. & $\%$ & \\
\hline$F$ & 2 & $3.85 \%$ & 0 & $0.00 \%$ & 1 & $1.92 \%$ & 0 & $0.00 \%$ & 0 & $0.00 \%$ & 3 & $5.77 \%$ & $0.001(\mathrm{HS})$ \\
\hline FG & 1 & $1.92 \%$ & 7 & $13.46 \%$ & 8 & $15.38 \%$ & 10 & $19.23 \%$ & 0 & $0.00 \%$ & 26 & $50.00 \%$ & \\
\hline G & 1 & $1.92 \%$ & 0 & $0.00 \%$ & 1 & $1.92 \%$ & 4 & $7.69 \%$ & 0 & $0.00 \%$ & 6 & $11.54 \%$ & \\
\hline $\mathrm{HI}$ & 0 & $0.00 \%$ & 0 & $0.00 \%$ & 1 & $1.92 \%$ & 6 & $11.54 \%$ & 1 & $1.92 \%$ & 8 & $15.38 \%$ & \\
\hline | & 0 & $0.00 \%$ & 1 & $1.92 \%$ & 3 & $5.77 \%$ & 2 & $3.85 \%$ & 3 & $5.77 \%$ & 9 & $17.31 \%$ & \\
\hline Total & 4 & $7.69 \%$ & 8 & $15.38 \%$ & 14 & $26.92 \%$ & 22 & $42.31 \%$ & 4 & $7.69 \%$ & 52 & $100.00 \%$ & \\
\hline
\end{tabular}


Table 3 Stages of modified MP3 compared with the stages of DI (male)

\begin{tabular}{|c|c|c|c|c|c|c|c|c|c|c|c|c|c|}
\hline \multirow{2}{*}{$\begin{array}{l}\text { DI } \\
\text { MP3 }\end{array}$} & \multicolumn{2}{|l|}{ D } & \multicolumn{2}{|l|}{$E$} & \multicolumn{2}{|l|}{$\mathrm{F}$} & \multicolumn{2}{|l|}{ G } & \multicolumn{2}{|l|}{$\mathrm{H}$} & \multicolumn{2}{|c|}{ Total } & \multirow[t]{2}{*}{$P$ Value } \\
\hline & No. & $\%$ & No. & $\%$ & No. & $\%$ & No. & $\%$ & No. & $\%$ & No. & $\%$ & \\
\hline$F$ & 6 & $12.50 \%$ & 1 & $2.08 \%$ & 1 & $2.08 \%$ & 1 & $2.08 \%$ & 0 & $0.00 \%$ & 9 & $18.75 \%$ & $<0.001(\mathrm{HS}$ \\
\hline FG & 1 & $2.08 \%$ & 8 & $61.67 \%$ & 3 & $6.25 \%$ & 3 & $6.25 \%$ & 0 & $0.00 \%$ & 15 & $31.25 \%$ & \\
\hline G & 0 & $0.00 \%$ & 0 & $0.00 \%$ & 1 & $2.08 \%$ & 3 & $6.25 \%$ & 0 & $0.00 \%$ & 4 & $8.33 \%$ & \\
\hline $\mathrm{H}$ & 0 & $0.00 \%$ & 0 & $0.00 \%$ & 0 & $0.00 \%$ & 2 & $4.17 \%$ & 0 & $0.00 \%$ & 2 & $4.17 \%$ & \\
\hline $\mathrm{HI}$ & 0 & $0.00 \%$ & 0 & $0.00 \%$ & 2 & $4.17 \%$ & 6 & $12.50 \%$ & 3 & $6.25 \%$ & 11 & $22.92 \%$ & \\
\hline | & 0 & $0.00 \%$ & 2 & $4.17 \%$ & 1 & $2.08 \%$ & 2 & $4.17 \%$ & 2 & $4.17 \%$ & 7 & $14.58 \%$ & \\
\hline Total & 7 & $14.58 \%$ & 11 & $22.92 \%$ & 8 & $16.67 \%$ & 17 & $35.42 \%$ & 5 & $10.42 \%$ & 48 & $100.00 \%$ & \\
\hline
\end{tabular}

Chi-square: 48.490 . DF: 20 . The $P$ value is 0.0004

$D /$ Demirjian's Index, MP3 middle phalanx of the third finger

radiation exposure point etc. make it a practical technique for the dental professional for the growth assessment.

The current study revealed a positive correlation between mandibular permanent second molar and modified MP3 which was in accordance to studies done by Vijayashree et al. (Vijayashree et al. 2014), in a South Indian population, who found highly positive correlation between DI of mandibular second molars and CVMI modified method. Perinetti et al. (Perinetti et al. 2011), analyzed the diagnostic performance of the circumpubertal dental maturation phases for the identification of individual-specific skeletal maturation phases and found that the dental and skeletal maturity are highly correlated, although the diagnostic performance of dental maturity for the identification of any stage of skeletal maturity is limited. Uysal et al. (Uysal et al. 2004), investigated the relationships between the stages of calcification of various teeth and skeletal maturity stages among
Turkish subjects and suggested that tooth calcification stages from panoramic radiographs might be clinically useful as a maturity indicator of the pubertal growth period. According to them, it is appropriate to put these skeletal and dental maturation relationships into daily orthodontic diagnostic practice, when treating a patient. Kumar et al. (Kumar et al. 2011), in a north western Indian population found a highly significant association exists between DI and CVMI. According to them, mandibular second molar DI stages are reliable indicators of skeletal maturity. Krailassiri et al. (Krailassiri et al. 2002), in a Thailand population found that tooth calcification stages from panoramic radiographs might be clinically useful as a maturity indicator of the pubertal growth period. Rai et al. (Rai and Anand 2007), investigated the relationship between the stage of calcification of various teeth and skeletal maturity stage among Indians and suggested that tooth calcification stages from panaromic radiographs might be clinical useful as a maturity

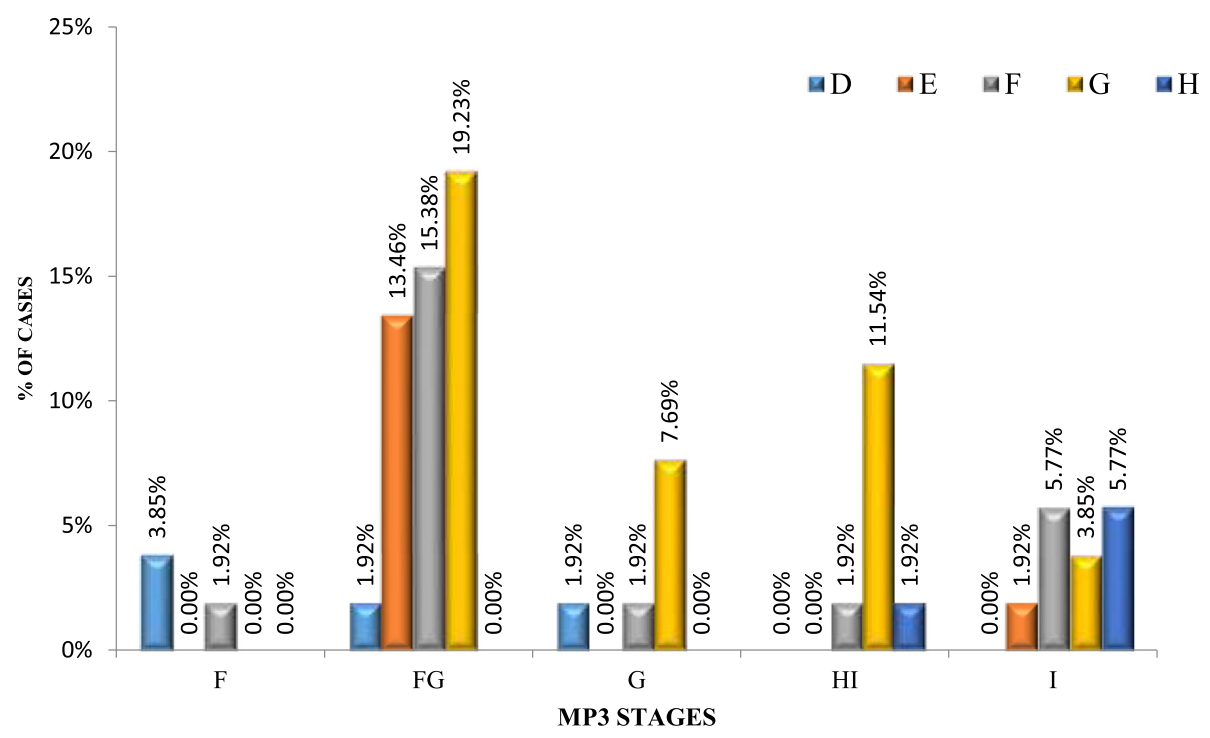

Fig. 3 Correlation between stages of modified MP3 and mandibular second molar in female 


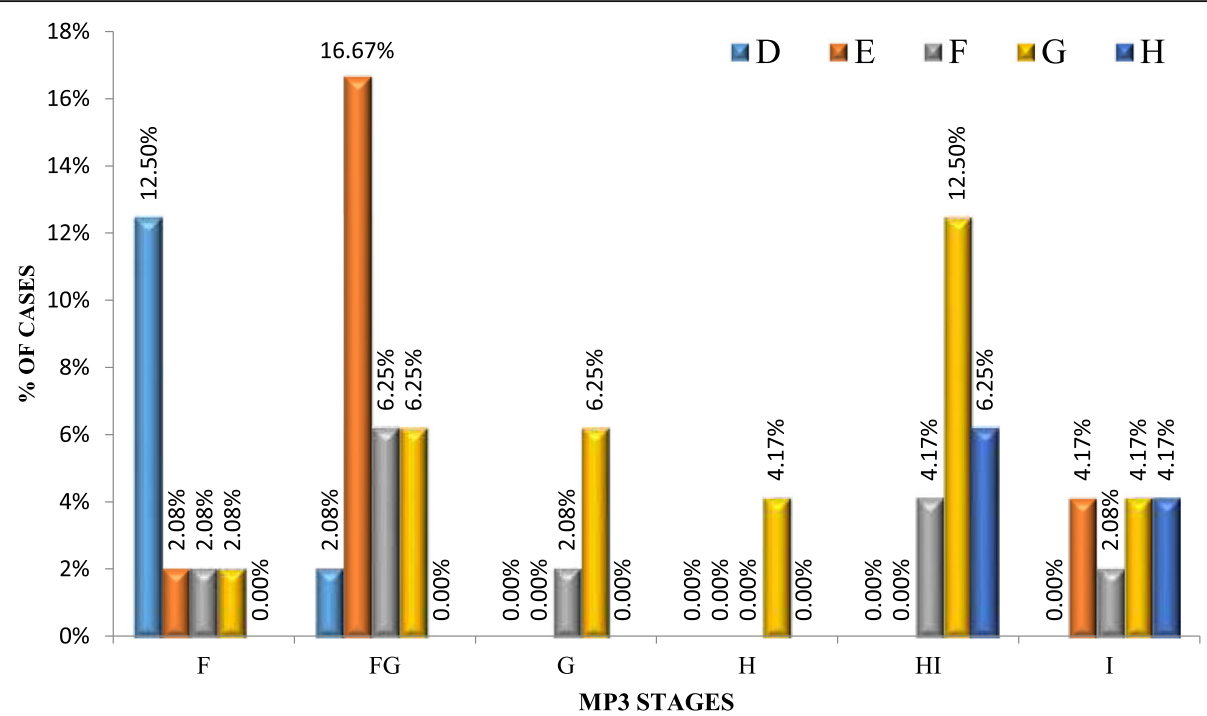

Fig. 4 Correlation between stages of modified MP3 and mandibular second molar in male

indicator after pubertal growth period, despite them using either mandibular canine, premolars or second molars in their studies.

The unique and significant findings from the present study imply that the stages of mandibular second molar calcification as observed on panoramic radiographs provide fairly accurate results and can be considered reliable indicators of skeletal maturity with the methodology suggested by Demirjian et al. (Demirjian et al. 1973).

\section{Conclusion}

The present study revealed a positive correlation between the calcification stages of mandibular second molar according to Demirjian Index and modified MP3 method proposed by Rajagopal and Kansal. It can be concluded that:

- Stage D and E of DI with the root length equal to or greater than the crown height, corresponds to F and FG stage of modified MP3 indicating the start of peak in mandibular growth which would be appropriate time to plan for functional appliances.

- Stage G and H of DI with partially to completely closed apex corresponding to modified MP3 HI and I indicates that peak of mandibular growth has

Table 4 Correlation of modified MP3 with DI

\begin{tabular}{llllll}
\hline Gender & Sample variable & Spearman $R$ & $\mathrm{t}(\mathrm{n}$-2) & p-level & Significance \\
\hline Male & MP3 with DI & 0.627 & 46 & $0.001^{*}$ & HS \\
Female & MP3 with DI & 0.441 & 50 & $0.01 *$ & $\mathrm{~S}$ \\
\hline
\end{tabular}

*Highly Significant Spearman's correlation

Spearman's correlation HS: Highly Significant

DI Demirjian's Index, MP3 middle phalanx of the third finger already occurred and is not appropriate for functional appliances.

The significant findings from the present study indicate that mandibular second molar calcification stages are reliable as skeletal maturity indicators, which can be used to predict the growth status in determining the optimal treatment timing.

\section{Abbreviations}

CVM: Cervical vertebrae maturation; DF: Degree of freedom; DI: Demirjian's index; MP3: Middle phalanx of the third finger; N: Total number; $p$ value: Probability value; SD: Standard deviation; SMI: Skeletal maturity indicator; SPSS: Statistical package for social sciences

\section{Acknowledgements}

The authors would like to acknowledge the timely help of faculty of Department of Oral and Maxillofacial Radiology, Pacific Dental College and Hospital Udaipur, india.

\section{Availability of data and materials}

Data sharing not applicable to this article as radiographs of orthodontics patients were retrieved from patient's data files.

\section{Authors' contributions}

MJ: Planned the paper, collected the data, wrote several sections and checked and revised all drafts and the final version. DR: Rechecked the collected data, wrote several sections and checked and revised all drafts and the final version. SA: wrote several sections, assisted in revision of the data, helped in the final version. SP: Helped in a statistical analysis, and wrote several sections in the manuscript. SF: wrote several sections and checked and revised all drafts and the final version of the manuscript. All the author and coauthors contributed significantly in preparing the manuscript. Authors are willing for transfer of copyright in case of acceptance of the article for publication. All authors read and approved the final manuscript.

\section{Ethics approval and consent to participate}

Ethical clearance was obtained from the Ethical Committee, Pacific Dental College and Hospital, Udaipur, India, and copy of the same has been attached. (Ref. No - PDCH/16/EC-14). 


\section{Consent for publication}

The manuscript does not contains any individual person's data in any form (including individual details, images or videos) other than radiographs collected with permission for the study.

\section{Competing interests}

The authors declare that they have no competing interests.

\section{Publisher's Note}

Springer Nature remains neutral with regard to jurisdictional claims in published maps and institutional affiliations.

Received: 13 February 2018 Accepted: 15 May 2018

Published online: 22 May 2018

\section{References}

Baccetti T, Franchi L, McNamara JA (2005) The cervical vertebral maturation (CVM) method for the assessment of optimal treatment timing in dentofacial orthopedics. Semin Orthod 11(3):119-129

Demirjian A, Goldstein H, Tanner J (1973) A new system of dental age assessment. Human Biol 45:211-227

Engström C, Engström H, Sagne S (1983) Lower third molar development in relation to skeletal maturity and chronological age. Angle Orthod 53(2): 97-106

Fishman LS (1982) Radiographic evaluation of skeletal maturation: a clinically oriented method based on hand-wrist films. Angle Orthod 52(2):88-112

Flores-Mir C, Burgess CA, Champney M, Jensen RJ, Pitcher MR, Major PW (2006) Correlation of skeletal maturation stages determined by cervical vertebrae and hand-wrist evaluations. Angle Orthod 76(1):1-5

García-Fernandez P, Torre H, Flores L, Rea J (1998) The cervical vertebrae as maturational indicators. J Clin Orthod 32(4):221-225

Grave K, Brown T (1976) Skeletal ossification and the adolescent growth spurt. Am J Orthod 69(6):611-619

Hägg U, Taranger J (1982) Maturation indicators and the pubertal growth spurt. Am J Orthod 82(4):299-309

Hegde G, Hegde N, Anil KK (2014) A new system for assessment of growth using mandibular canine calcification stages and its correlation with modified MP3 stages. J Pharm Bioallied Sci 6(Suppl 1):S58

Houston W, Miller J, Tanner J (1979) Prediction of the timing of the adolescent growth spurt from ossification events in hand-wrist films. Br J Orthod 6(3): 145-152

Krailassiri S, Anuwongnukroh N, Dechkunakorn S (2002) Relationships between dental calcification stages and skeletal maturity indicators in Thai individuals. Angle Orthod 72(2):155-166

Kumar S, Roy AS, Garg A, Hamid SB, Tyagi S, Kumar A (2017) Correlation between maxillary canine calcification and skeletal maturation. J Clin Diagn Res 11(5):ZC13

Kumar S, Singla A, Sharma R, Virdi MS, Anupam A, Mittal B (2011) Skeletal maturation evaluation using mandibular second molar calcification stages. Angle Orthod 82(3):501-506

Nolla CM (1960) The development of the permanent teeth. J Dent Child 27: 254-263

Perinetti G, Contardo L, Gabrieli P, Baccetti T, Di Lenarda R (2011) Diagnostic performance of dental maturity for identification of skeletal maturation phase. Eur J Orthod 34(4):487-492

Rai B, Anand S (2007) Relationship of different radiograph: maturity indicator. Adv In Med Dent Sci 1(1):115-19

Rai B, Kaur J, Anand S, Jain R, Sharma A, Mittal S (2008) Accuracy of the Demirjian method for the Haryana population. Internet J Dent Sci 6(1):1-4

Rajagopal R, Kansal S (2002) A comparison of modified MP3 stages and the cervical vertebrae as growth indicators. J Clin Orthod 36(7):398

Reddy W, Prasad GD, Hirey RK, Kumar SS, Krishnan KS (2014) A comparison of skeletal maturation assessed from MP3 and its correlation with dental maturation. J Ind Orthod Soc 48(5):325

Şahin Sağlam AM, Gazilerli Ü (2002) The relationship between dental and skeletal maturity. J Orofac Orthop 63(6):454-462

Uysal T, Sari Z, Ramoglu SI, Basciftci FA (2004) Relationships between dental and skeletal maturity in Turkish subjects. Angle Orthod 74(5):657-664

Van der Linden F, Wassenberg H, Bakker P (1979) The transition process in retrospect. Inf Orthod Kieferorthop 11(3):325
Vijayashree U, Pai V, Naik VR (2014) Second molar calcification stages to evaluate skeletal maturation: a cross-sectional radiographic study. APOS Trends Orthod 4(6):156

Yadav V, Loomba A, Autar R (2017) A comparative evaluation of dental calcification stages and skeletal maturity indicators in north-Indian children. Natl J Maxillofac Surg 8(1):26

\section{Submit your manuscript to a SpringerOpen ${ }^{\circ}$ journal and benefit from:}

- Convenient online submission

- Rigorous peer review

- Open access: articles freely available online

- High visibility within the field

Retaining the copyright to your article

Submit your next manuscript at springeropen.com 